\title{
Studying The Association Of Metformin Dose With Peripheral Neuropathy In Diabetic Patients At Jordanian Royal Medical Services
}

\author{
Wafa'a Jamil abu Jama'ah \\ Motazbellah Fat'hi Alzu'bi \\ Alaa Rfeefan Alabbadi \\ Alaa Mohammed Al Mrayat \\ Ola Ahmad Aldmour \\ Jaafar Abu Abeeleh \\ Royal Medical Services, Jordan
}

Ahed $J$ Alkhatib

Jordan University of Science and Technology, Jordan

doi: 10.19044/esj.2016.v12n6p22 URL:http://dx.doi.org/10.19044/esj.2016.v12n6p22

\begin{abstract}
The aim of the present study was to study the association of metformin dose with peripheral neuropathy in diabetic patients at Jordanian Royal Medical Services. A retrospective study was conducted to collect data from files of 392 diabetic patients at Jordanian Royal Medical Services. A working sheet was created for each patient that included the following information: gender, age, peripheral neuropathy, vitamin B12 status, metformin use, and metformin dose. Data were analyzed using SPSS version 20. Data were represented as frequencies and percentages for categorical variables such as gender, and vitamin B12 status; means and standard deviations for continuous variables such as age. The relationships between variables were examined using Chi-Square, and One Way Anova. Significance was considered at alpha level $<0.05$. Study findings showed that the prevalence of diabetic peripheral neuropathy among participants was about 43\%. Vitamin B12 deficiency was about 7\%. Diabetic peripheral neuropathy was significantly associated with gender $(\mathrm{p}=0.018)$. No significant associations were observed between peripheral neuropathy and vitamin B12 or metformin dose. Taken together, the results of the present study showed that treatment with metformin did not lead to vitamin B12 deficiency, although the prevalence of diabetic peripheral neuropathy was relatively high (about $43 \%$ ).
\end{abstract}


Keywords: Peripheral neuropathy, diabetes, vitamin B12, metformin

\section{Introduction}

Type 2 diabetes mellitus (DM) is considered as one of the most prevalent metabolic diseases at global level, and its prevalence is likely to with age and obesity (Butterfield et al., 2014). The functions of several organs are deteriorated by DM such as brain (Arnold et al., 2014).

Metformin is used widely as anti-diabetic drug (Ruggiero-Lopez et al., 1999; Kirpichnikov etal.,2002; Yamagishi et al., 2008; Ma et al., 2015). It acts through lowering glucose hepatic production (Derosa et al., 2008). Although the mechanisms of metformin action are not well clear, it has been recently suggested that to some pleiotropic actions (Chen et al., 2001). Among these actions is AMP-activated protein kinase (AMPK) (Steinberg and Kemp, 2009). Several roles of AMPK have been involved in DM, neuroprotection, anti-inflammation and alteration of oxidative stress (Chakraborty et al., 2011; Salminen et al., 2011).

It has been indicated to the possibility of inhibition of injury-induced neuropathic pain (Melemedjian etal.,2011). In another study, metformin was able to reverse mechanical allodynia in lumbar radiculopathy pain in experimental models such as rats and mice (Taylor et al.,2013). Furthermore, using mouse model showed the potential of metformin to offer protection against chemotherapy induced neuropathic pain (Mao-Yingetal.,2014).

Other studies showed that long term treatment by metformin lowers serum concentration of cyanocobalamine up to 30\%, and this may occur in approximately in 30\% of treated patients (Mazokopakis and Starakis, 2012). This process depends on inhibition of cyanocobalamine intrinsic factor complex absorption, which depends on calcium, so that it can be treated with calcium supplementation (Jager et al., 2010). It has been demonstrated that the treatment of metformin is not likely to cause megaloblastic anemia except in cases of heavily doses over a long duration (Mazokopakis and Starakis, 2012).

\section{Study objectives}

To study the association of metformin dose with peripheral neuropathy in diabetic patients at Jordanian Royal Medical Services.

\section{Methods and subjects}

This section includes the methods of choice which were involved to collect data and to conduct the appropriate statistical analyses. 


\section{Study design and setting}

A retrospective design was employed to collect data from study participants. The study was conducted at Jordanian Royal Medical Services.

\section{Study sample}

Study sample included a total of 392 diabetic patients.

\section{Data collection}

A working sheet for each diabetic patient was created for each diabetic patient. The required information were extracted from files of patients including age, gender, peripheral neuropathy, metformin use, metformin dose, and the status of vitamin B12.

\section{Data analysis}

All data were entered into excel sheet to obtain the raw data. SPSS version 20 was used to analyze data. Categorical variables such as gender were presented as frequencies and percentages, while continuous variables such as age was presented as mean and standard deviation. The relationships between variables were examined using Chi-Square, $\mathrm{T}$ test, and One Way Anova. Significance was considered at alpha level $\leq 0.05$.

\section{Results}

\section{General characteristic of participants}

As it can be demonstrated in table 1, the mean age of study participants was 56.18+9.51 years. About 47\% were males. The prevalence of peripheral neuropathy was approximately 43\%. About $85 \%$ of diabetic patients received metformin treatment, and the mean metformin dose was 1474.68+906.78 mg/day. Vitamin B12 deficiency was shown among about $7 \%$ of patients.

\begin{tabular}{cc}
\multicolumn{2}{c}{ Table 1: General characteristic of participants } \\
Variable & Description \\
Age (M+SD) years & $56.18 \pm 9.51$ \\
Gender (N, \%): & $183(46.7 \%)$ \\
Males & $209(53.3 \%)$ \\
Females & \\
Peripheral neuropathy(N, \%): & $168(42.9 \%)$ \\
Yes & $224(57.1 \%)$ \\
No & \\
Metformin (N, \%): & $331(84.6 \%)$ \\
Yes & $61(15.6 \%)$ \\
No & $1474.68 \pm 906.78$ \\
Metformin dose (M+SD) mg & \\
Vitamin B12 (N, \%): & $26(6.6 \%)$ \\
Deficiency & $366(93.4 \%)$ \\
Normal
\end{tabular}




\section{The relationship between peripheral neuropathy and study variables}

As it can be seen in table 2, male were more likely to develop peripheral neuropathy than females, and this variation was statistically significant $(\mathrm{p}=0.018)$. No significant variations were observed between peripheral neuropathy and each of metformin use $(\mathrm{p}=0.780)$ and vitamin $\mathrm{B} 12$ status $(\mathrm{p}=0.446)$.

Table 2: The relationship between peripheral neuropathy and study variables

Variable

Gender
Males
Females
Metformin use
No
Yes
Vitamin B12
Normal
Deficiency

Peripheral neuropathy

\begin{tabular}{c|c|c|c}
\hline \multicolumn{3}{|c|}{ Yes } & \multicolumn{1}{c}{ No } \\
\hline $\mathrm{N}$ & $\%$ & $\mathrm{~N}$ & $\%$ \\
\hline 90 & 49.2 & 93 & 50.8 \\
78 & 37.3 & 131 & 62.7 \\
143 & 43.2 & 188 & 56.8 \\
25 & 41 & 36 & 59 \\
13 & 50 & 13 & 50 \\
155 & 42.3 & 211 & 57.7
\end{tabular}

$\mathrm{P}$ value

0.018

0.780

0.446

\section{The effects of study variables on peripheral neuropathy}

In this section, One Way Anova was conducted to examine the effects of study variables on peripheral neuropathy. As it can be illustrated in table 3, the following variables were examined: age, gender, metformin, metformin dose, and vitamin B2. Among these variables, gender was significantly associated with peripheral neuropathy $(\mathrm{p}=0.018)$, while the remaining variables were not statistically associated significantly with peripheral neuropathy $(\mathrm{p}>0.05)$.

Table 3: The effects of study variables on peripheral neuropathy

$\begin{array}{cccccc}\text { Variable } & \text { Sum of squares } & \text { DF } & \text { Mean square } & \text { F } & \text { P-value } \\ \text { Age } & 46.14 & 1 & 46.164 & 0.510 & 0.476 \\ \text { Gender } & 1.395 & 1 & 1.395 & 5.66 & 0.018 \\ \text { Metformin } & 0.014 & 1 & 0.014 & 0.103 & 0.748 \\ \text { Metformin } & 368333.466 & 1 & 368333.466 & 0.447 & 0.504 \\ \text { dose } & & & & & \\ \text { Vitamin B12 } & .036 & 1 & .036 & 0.578 & 0.448\end{array}$

\section{Discussion}

The present study showed that the prevalence of peripheral neuropathy among diabetic patients was about $43 \%$. The prevalence of peripheral neuropathy in other studies such as the study of Davies et al (2006) in which the prevalence of peripheral neuropathy was $26.4 \%$. other studies reported the prevalence of peripheral neuropathy to vary from 3\% (Sorensen, Molyneaux and, Yue, 2002) to 32\% (Ziegler etal., 1993). 
The findings of our study showed that vitamin B12 deficiency was about 93\% among diabetic patients. Reviewing the literature showed that the prevalence of vitamin B12 deficiency varied from 5.8\% to 33\% (Pflipsen et al., 2009; Qureshi, Ainsworth, and Winocour, 2011; Reinstatler et al., 2012).

The findings of the present study revealed a significant role of gender to develop peripheral neuropathy $(p=0.018)$. This finding is not consistent with the results of the study of Kodali, Seshaiah, and Kumar (1990) who found higher prevalence of peripheral neuropathy among women compared with men.

No significant association was found between diabetic peripheral neuropathy and vitamin B12 deficiency and metformin ( $\mathrm{p}>0.05)$. We think, based on these findings, that metformin provides neuroprotection and confirm other studies such as Chakraborty et al (2011) and; Salminen et al (2011).

\section{Conclusion}

The results of the present study showed that treatment with metformin does not lead to vitamin B12 deficiency, although the prevalence of diabetic peripheral neuropathy was relatively high (about 43\%). Diabetic peripheral neuropathy was significantly associated with gender $(p=0.018)$.

\section{References:}

A. Chakraborty, S. Chowdhury, M. Bhattacharyya (2011). Effect of metformin on oxidative stress, nitrosative stress and inflammatory biomarkers in type 2 diabetes patients, Diabetes Res. Clin. Pract. 93, 56-62.

A. Salminen, J.M. Hyttinen, K. Kaarniranta (2011). AMP-activated protein kinase inhibits NF kappa B signaling and inflammation: impact on health span and lifespan, J. Mol. Med. (Berl.) 89, 667-676.

Chen Y, Shen X, Fenyk-Melody J, Wu M, Ventre J, Doebber T, et al (2001). Role of AMP-activated protein kinase in mechanism of metformin action. $\mathrm{J}$ Clin Invest, 108:1167-1174.

D. Ruggiero-Lopez, M. Lecomte, G. Moinet, G. Patereau, M. Lagarde, N. Wiernsperger (1999). Reaction of metformin with dicarbonyl compounds. Possible implication in the inhibition of advanced glycation end product formation. Biochem. Pharmacol. 58, 1765-1773.

D.A. Butterfield, F. Di Domenico, E. Barone (2014). Elevated risk of type 2 diabetes for development of Alzheimer disease: a key role for oxidative stress in brain, Biochim. Biophys. Acta 1842, 1693-1706.

G.R. Steinberg, B.E. Kemp (2009). AMPK in health and disease, Physiol. Rev. 89, 1025-1078.

Giuseppe Derosa, Sibilla A.T. Salvadeo, Angela D’Angelo, Elena Fogari, Pietro D. Ragonesi, Leonardina Ciccarelli, Mario N. Piccinni, Ilaria Ferrari, 
Alessia Gravina, Pamela Maffioli, Arrigo F. Cicero (2008). Rosiglitazone therapy improves insulin resistance parameters in overweight and obese diabetic patients intolerant to metformin. Archives of Medical Research, 39, 412-419.

Jager J, Kooy A, Lehert P, Wulffelé MG, van der Kolk J, Bets D, et al (2010). Long term treatment with metformin in patients with type 2 diabetes and risk of vitamin B-12 deficiency: randomized placebo controlled trial. BMJ, 340: c2181.

Junxiong Ma, Hailong $\mathrm{Yu}$, Jun Liu,Yu Chen, Qi Wang, Liangbi Xiang (2015). Metformin attenuates hyperalgesia and allodynia in rats with painful diabetic neuropathy induced by streptozotocin. European Journal of Pharmacology,764, 599-606.

Kirpichnikov, D., McFarlane, S.I., Sowers, J.R (2002). Metformin: an update. Ann. Intern. Med.137,25-33.

Kodali VR, Seshaiah V, Kumar TV (1990). Gender differences in the associated complications among type-II diabetics with peripheral neuropathy. Neurologija., 39 (1):3-7.

Mao-Ying, Q.L., Kavelaars, A., Krukowski, K., Huo, X.J., Zhou, W., Price, T.J., Cleeland, C., Heijnen, C.J (2014). The anti-diabetic drug metformin protects against che- motherapy-induced peripheral neuropathy in a mouse model. PLoS One 9, e100701.

Mark Davies, Sinead Brophy, Rhys Williams, Ann Taylor (2006). The Prevalence, Severity, and Impact of Painful Diabetic Peripheral Neuropathy in Type 2 Diabetes. Diabetes Care 29:1518 -1522.

Mazokopakis E, Starakis I (2012). Recommendations for diagnosis and management of metformin-induced vitamin B12 (Cbl) deficiency. Diabetes Res Clin Pract 2012;97:359-67.

Pflipsen M, Oh R, Saguil A, Seehusen D, Topolski R (2009). The Prevalence of Vitamin B12 Deficiency in Patients with Type 2 Diabetes: A Cross Sectional Study. J Am Board Fam Med, 22:528-34.

Qureshi S, Ainsworth A, Winocour P (2011). Metformin therapy and assessment for vitamin B12 deficiency: is it necessary? Practical Diabetes, 28:302-4.

Reinstatler L, Qi Y, Williamson R, Garn J, Oakley-Jr G (2012). Association of Biochemical B12 Deficiency With Metformin Therapy and Vitamin B12 Supplements. The National Health and Nutrition Examination Survey, 19992006. Diabetes Care, 35:327-33.

S. Yamagishi, K. Nakamura, T. Matsui, S. Ueda, K. Fukami, S. Okuda (2008). Agents that block advanced glycation end product (AGE)-RAGE (receptor for AGEs)-oxidative stress system: a novel therapeutic strategy for diabetic vascular complications. Expert Opin. Investig. Drugs, 17, 983-996. 
S.E. Arnold, I. Lucki, B.R. Brookshire, G.C. Carlson, C.A. Browne, H. Kazi, S. Bang, B.R. Choi, Y. Chen, M.F. McMullen, S.F. Kim (2014). High fat diet produces brain insulin resistance, synaptodendritic abnormalities and altered behavior in mice, Neurobiol. Dis. 67, 79-87.

Sorensen L, Molyneaux L, Yue DK (2002). Insensate versus painful diabetic neuropathy: the effects of height, gender, ethnicity and glycaemic control. Diabetes Res Clin Pract 57:45-51.

Taylor, A.,Westveld, A. H., Szkudlinska, M., Guruguri, P., Annabi, E., Patwardhan, A., Price, T. J., Yassine, H. N (2013).The use of metformin is associated with decreased lumbar radiculopathy pain. J. Pain Res. 6, 755763.

Ziegler D, Gries FA, Spuler M, Lessmann F (1993). The epidemiology of diabetic neuropathy: DiaCAN Multicenter Study Group. Diabet Med 10 (Suppl. 2):82S-86S. 